\title{
A puzzling periodicity in the pulsating DA white dwarf G 117-B15A
}

\author{
R. Kotak ${ }^{1,2}$, M. H. van Kerkwijk ${ }^{3,4}$, and J. C. Clemens ${ }^{5, \star \star}$ \\ 1 Lund Observatory, Box 43, 22100 Lund, Sweden \\ 2 Astrophysics Group, Imperial College London, Blackett Laboratory, Prince Consort Road, London, SW7 2BZ, UK \\ 3 Astronomical Institute, Utrecht University, PO Box 80000, 3508 TA Utrecht, The Netherlands \\ 4 Department of Astronomy and Astrophysics, University of Toronto, 60 St George Street, Toronto, Ontario M5S 3H8, Canada \\ e-mail: mhvk@astro.utoronto.ca \\ 5 Department of Physics and Astronomy, University of North Carolina, Chapel Hill, NC 27599-3255, USA \\ e-mail: clemens@physics.unc.edu
}

Received 30 June 2003 / Accepted 19 September 2003

\begin{abstract}
We present time-resolved optical spectrophotometry of the pulsating hydrogen atmosphere (DA) white dwarf G 117-B15A. We find three periodicities in the pulsation spectrum (215 s, $272 \mathrm{~s}$, and $304 \mathrm{~s})$ all of which have been found in earlier studies. By comparing the fractional wavelength dependence of the pulsation amplitudes (chromatic amplitudes) with models, we confirm a previous report that the strongest mode, at $215 \mathrm{~s}$, has $\ell=1$. The chromatic amplitude for the $272 \mathrm{~s}$ mode is very puzzling, showing an increase in fractional amplitude with wavelength that cannot be reproduced by the models for any $\ell$ at optical wavelengths. Based on archival HST data, we show that while the behaviour of the $215 \mathrm{~s}$ mode at ultra-violet wavelengths is as expected from models, the weird behaviour of the $272 \mathrm{~s}$ periodicity is not restricted to optical wavelengths in that it fails to show the expected increase in fractional amplitude towards shorter wavelengths. We discuss possible causes for the discrepancies found for the $272 \mathrm{~s}$ variation, but find that all are lacking, and conclude that the nature of this periodicity remains unclear.
\end{abstract}

Key words. stars: white dwarfs - stars: oscillations - stars: individual: G 117-B15A

\section{Introduction}

The hydrogen-atmosphere pulsating white dwarfs (DAVs or ZZ Cetis), occupy a narrow (1000 K wide) instability strip at $\sim 11.5 \mathrm{kK}$, and exhibit multi-periodic flux variations of several hundreds of seconds that are primarily due to changes in the effective temperature (Robinson et al. 1982). The DAVs can be roughly divided into the hotter objects that have relatively simple and stable pulsational spectra consisting of a few short period, low amplitude modes, and the cooler ones that have more modes in total, with generally longer periods and larger amplitudes, and show moderate to severe amplitude variability on several different timescales, with some modes even disappearing from one season to the next (e.g. Koester 2002).

In order to infer the interior properties of the white dwarf, the modes in any given pulsator must be individually

Send offprint requests to: R. Kotak, e-mail: rubina@ic.ac.uk

* Based in part on data obtained at the W.M. Keck Observatory, which is operated as a scientific partnership among the California Institute of Technology, the University of California and the National Aeronautics and Space Administration. The Observatory was made possible by the generous financial support of the W.M. Keck Foundation.

$\star \star$ Alfred P. Sloan Research Fellow. identified i.e. the spherical degree $(\ell$; number of nodal lines on the surface), the azimuthal order $(m)$, and the radial order ( $n$; number of nodes from centre to surface) have to be known with some confidence. While $\ell$ and $m$ can be determined observationally, $n$ has to be deduced by detailed comparison with pulsation models. The spherical degree can be inferred in a number of ways, none of which are completely reliable on their own. Most studies to date have relied on the rotationally-induced splitting of modes and/or the comparison of observed distribution of mode periods with predicted ones. Assuming spherical symmetry, frequencies of modes having the same $n$ and $\ell$ are degenerate. Slow rotation lifts this degeneracy resulting in $2 \ell+1$ split components. The observation of all split components is an indicator of the $\ell$ value of a mode. The other method relies on the expectation that in the absence of compositional boundaries and for large $n$, modes of consecutive radial order (and same $\ell$ ) are equally spaced in period.

Robinson et al. (1995) developed another method which relies on the increased importance of limb-darkening at short wavelengths and the effect this has on pulsation amplitudes as a function of wavelength. The advantage of this method is that the resulting variation of mode amplitudes with wavelength is a function of $\ell$ (but not $m$ ), thereby permitting 
mode-identification in the absence of a large number of modes or rotationally-split modes. We will focus on this latter method here.

G 117-B15A epitomises the hotter DAVs, and has been extensively observed since the discovery of its variability almost three decades ago (Richer \& Ulrych 1974; McGraw \& Robinson 1976). Its pulsation spectrum is relatively simple, with the dominant mode occurring at $215 \mathrm{~s}$. This mode has been shown to be remarkably stable in amplitude (Kepler et al. 2000a). Two other modes at 272 and 304 s also appear in all data sets, although they are weaker and show variations in amplitude. Other possible real modes have also been reported by Kepler et al. (1995). However, the three modes mentioned above appear in all data sets for this object. A Whole Earth Telescope (WET, Nather et al. 1990) run failed to reveal both a coherent set of splittings for any mode (Kepler et al. 1995), and a series of modes that could be identified with a particu$\operatorname{lar} \ell$ and consecutive $n$. A clear $\ell$ identification was therefore not possible.

On the other hand, ultra-violet light curves and quantitative use of model atmospheres allowed Robinson et al. (1995) to assign $\ell=1$ to the $215 \mathrm{~s}$ mode in G 117-B15A. Using this $\ell=1$ identification of the $215 \mathrm{~s}$ mode, and assuming an $\ell=1$ identifcation for the $272 \mathrm{~s}$ and $304 \mathrm{~s}$ modes, Bradley (1998) derived a range of parameters, from thickness of the superficial hydrogen layer to the core $\mathrm{C} / \mathrm{O}$ ratio, from fits to pulsation models.

The primary motivation for the work described in this paper stemmed from a need for independent confirmation of the $\ell$ identification of the $215 \mathrm{~s}$ mode, and the need for constraints on the $\ell$ values of the other modes. As we describe below, our data however, have revealed a number of surprises.

\section{Observations}

Time-resolved spectra of G 117-B15A were obtained using the Low Resolution Imaging Spectrograph (Oke et al. 1995) mounted at the Cassegrain focus of the Keck II telescope on 1997 December 11. We acquired a total of 235 frames, from 10:47:42 to 14:02:56 U.T. An 8.'7-wide slit was used together with a 600 line $\mathrm{mm}^{-1}$ grating covering the $3450-5960 \AA$ range at $1.25 \AA \mathrm{pixel}^{-1}$. The seeing was $1^{\prime \prime} .2$ and some patchy cirrus was present. The common proper motion companion was also accomodated in the slit. The reduction of the data included the usual procedures of bias subtraction, flat fielding, optimum extraction, and wavelength and flux calibration. Flux calibration was carried out with respect to the flux standard G 191-B2B. The use of a wide slit (for photometric accuracy) requires additional corrections to the wavelength scale for differential atmospheric refraction and wandering in the slit. These were performed in a manner identical to that described in Kotak et al. (2002).

To complement the optical data, we retrieved archival data taken at ultra-violet wavelengths with the High Speed Photometer on-board the Hubble Space Telescope (see Robinson et al. 1995). The target was observed four times with the F184W filter $\left(\lambda_{\text {eff }}=1920 \AA, F W H M=360 \AA\right)$ and twice with the F145M filter $\left(\lambda_{\text {eff }}=1570 \AA, F W H M=215 \AA\right)$.

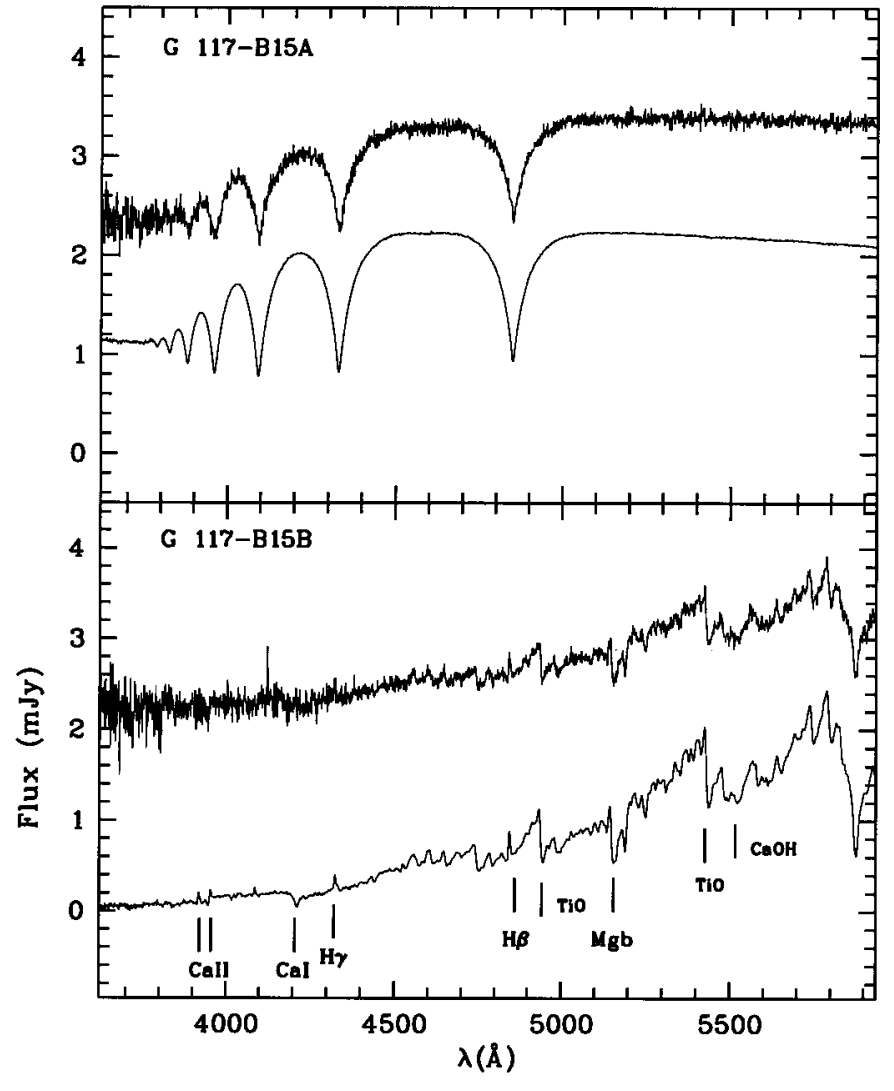

Fig. 1. Sample and average spectra of G 117-B15A (top panel) and its common proper motion companion G 117-B15B (bottom panel). The sample spectrum of $\mathrm{G} 117-\mathrm{B} 15 \mathrm{~A}$ is offset from the mean spectrum by $+1.7 \mathrm{mJy}$ while that of $\mathrm{G} 117-\mathrm{B} 15 \mathrm{~B}$ is offset by $+2.2 \mathrm{mJy}$. The average spectra of G 117-B15A and G 117-B15B have been scaled such that the fluxes at $\sim 5500 \AA$ correspond to the published $V$ band magnitudes $(V=+15.54$ and +16.06 respectively $)$.

\section{Average spectra}

Sample and average spectra of G 117-B15A,B are shown in Fig. 1. As expected, the spectrum of $G$ 117-B15A shows Balmer lines only. The spectrum of the common proper motion companion, G 117-B15B, shows strong TiO bands indicating that its spectral type is later than about M0. The TiO band head at $\sim 4750 \AA$ is not particularly well-developed due to a blend with the $\mathrm{MgH} \lambda 4780 \AA$ feature, suggesting a spectral type of M3V-M4V. The presence of the $\mathrm{CaOH}$ band and the absence of the Fe I $\lambda 4384 \AA$ line also suggests a spectral type of $\mathrm{M} 3 \mathrm{~V}-\mathrm{M} 5 \mathrm{~V} . \mathrm{H} \beta, \mathrm{H} \gamma$, and possibly $\mathrm{H} \delta$ are seen in emission as are the weak $\mathrm{Ca}$ II $\mathrm{H}$ and $\mathrm{K}$ lines. We tentatively assign a spectral type of M3Ve.

Attempting to match the flux at $\sim 5500 \AA$ and the slope of the spectrum using the NextGen models described in Hauschildt et al. (1999), we obtain a reasonable match for $T_{\text {eff }} \sim 3.4 \mathrm{kK}$ and $\log g \sim 4.5$ with somewhat higher metallicity models, $[M / H]=0.5$, providing a slightly better match than models computed with solar metallicity i.e. $[M / H]=0.0$. 


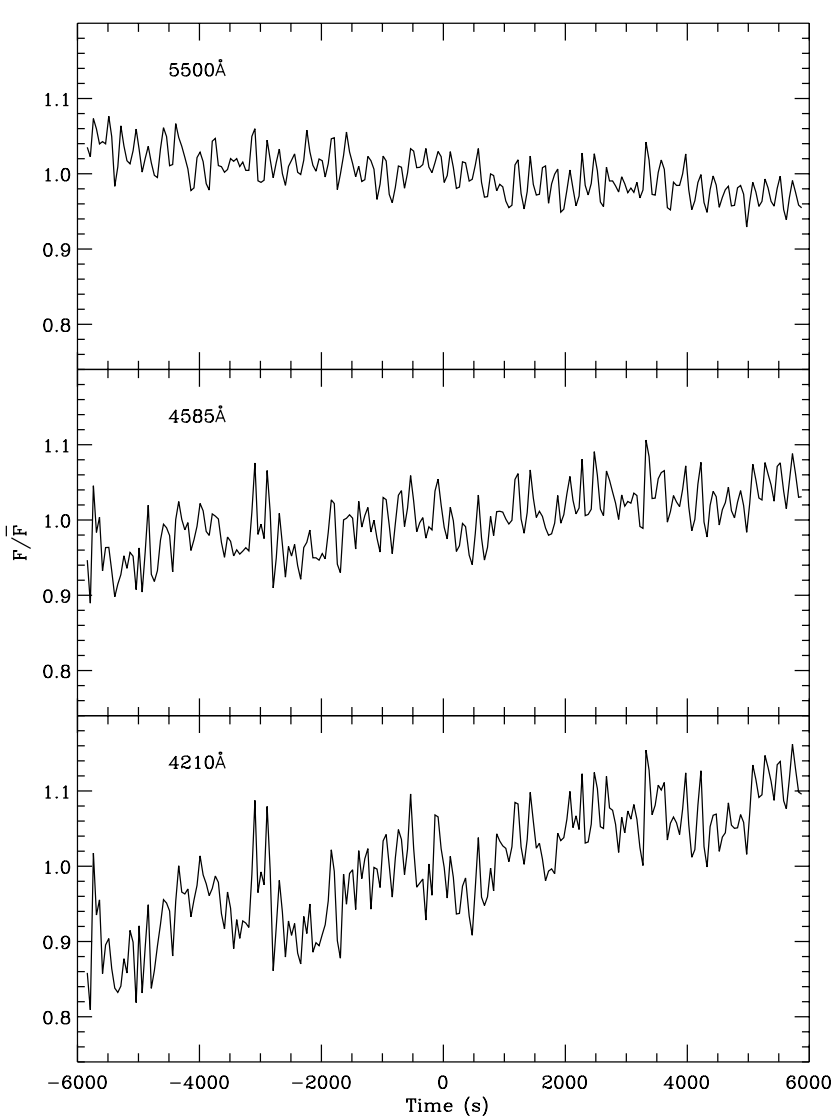

Fig. 2. Light curves constructed from the optical spectra. The times shown are relative to the middle of the time series.

\section{Periodicities in the light curves}

Optical light curves were constructed by dividing the linefree region of the continuum between $\sim 5300-5700 \AA$ (longward of $\mathrm{H} \beta$ ), $\sim 4400-4800 \AA$ (between $\mathrm{H} \gamma$ and $\mathrm{H} \beta$ ), and $\sim 4000-4300 \AA$ (between $\mathrm{H} \epsilon$ and $\mathrm{H} \gamma$ ) by a portion of the spectrum of the companion stretching from $\sim 4150-5470 \AA$. This was done in order to cancel out atmospheric fluctuations. We preferred the above procedure rather than dividing each chosen region of $\mathrm{G}$ 117-B15A by an exactly equivalent region of $\mathrm{G} 117-\mathrm{B} 15 \mathrm{~B}$ as there is very little flux from $\mathrm{G} 117-\mathrm{B} 15 \mathrm{~B}$ shortward of $\sim 4000 \AA$. This procedure does, however, introduce a slight linear trend in the light curves as a result but this is easily accounted for by including a first-order polynomial in the fit of the light curves as described below.

Figure 3 shows the Fourier Transforms of the normalised light curves for both the three optical and ultra-violet datasets, and the residuals after fitting the sum of three sinusoids of the form $A(\cos 2 \pi f t-\phi)$. Here, $A$ denotes the amplitude, $f$ the frequency, and $\phi$ the phase. As mentioned earlier, a low order polynomial was also included in the fit. The frequencies were fixed to those reported by Kepler et al. (1995) from WET data as these are more precise. Given their higher frequency resolution, Kepler et al. (1995) found several peaks in each of the 215,270 , and $304 \mathrm{~s}$ regions. For each of our three peaks, our choice of periods from their Table 2 was based on the one that resulted in the best fit of our light curve(s). For two of the peaks, viz. 215 and $304 \mathrm{~s}$, these also corresponded to the ones to which

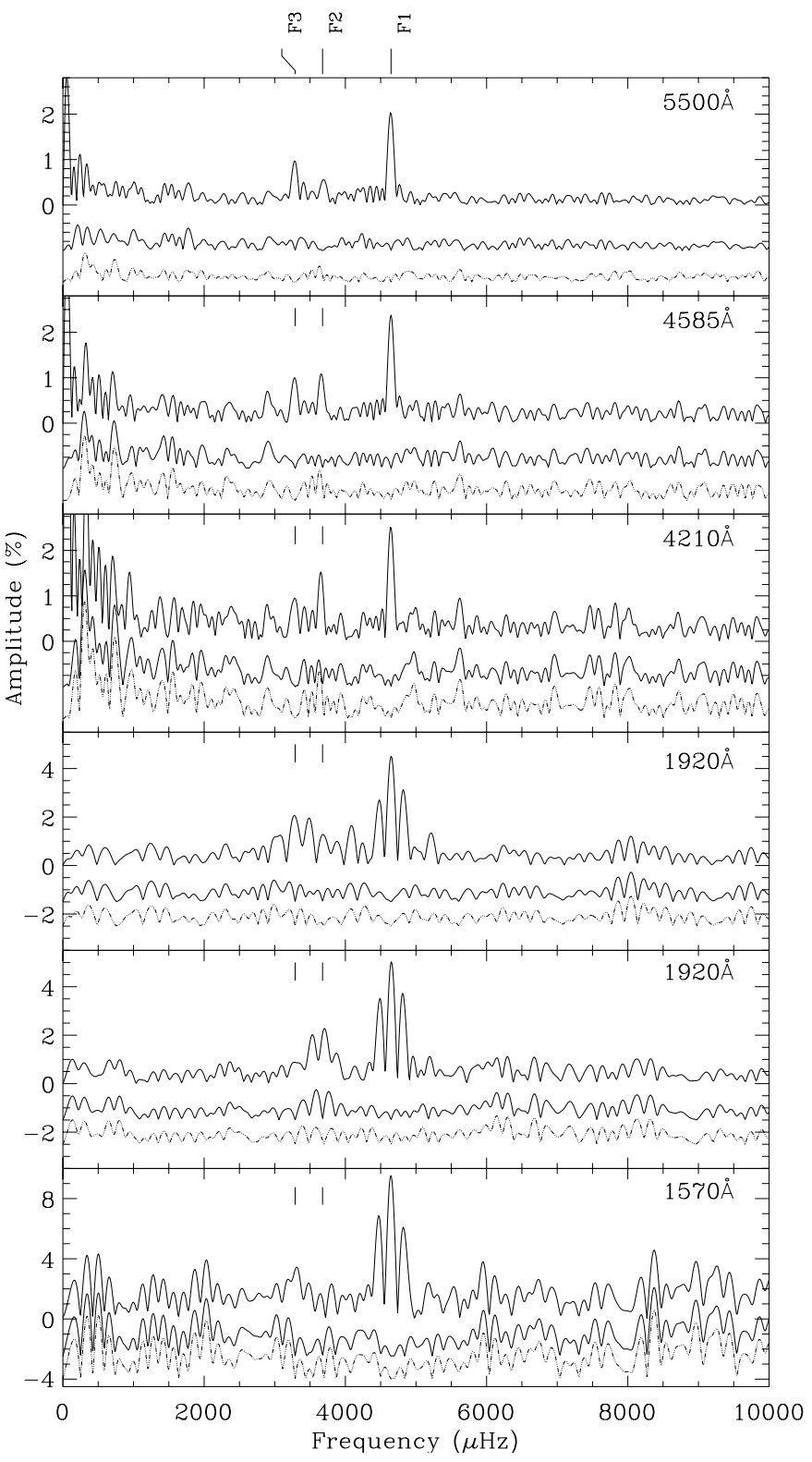

Fig. 3. Fourier Transforms (FTs) of the light curves for data taken in different passbands, with the approximate central wavelengths shown in the top righthand corner of each panel. The top three panels show the FTs from our optical dataset while the lower three show the FTs from archival UV data. The middle curve in each panel shows the residuals after fitting sinusoids with frequencies listed in Table 1 . These are offset by $-1 \%$ in the top three panels, by $-1.5 \%$ in the next two panels, and by $-2.5 \%$ in the lowermost panel. The dash-dot-dot-dashed (lowermost) curve in all panels shows the residuals (offset by $-1.5 \%$ in the top three panels, by $-2.5 \%$ in the next two panels, and by $-4 \%$ in the lowermost panel) obtained by including a periodicity at $270.455 \mathrm{~s}$ instead of one at $271.95 \mathrm{~s}$ (F2). Figure 4 shows an expanded view. Larger residuals result in every case. The positions of F2 and F3 are marked in all panels. Note the differing scales of the vertical axes of the two sets of triplots.

they attached the highest significance. We therefore fixed these to $215.1968 \mathrm{~s}$ and $304.052 \mathrm{~s}$. For the peak around $272 \mathrm{~s}$, we found that the $271.95 \mathrm{~s}$ peak matched our light curves better than the one at 270.455 s (see Fig. 4 and the lowermost curve 


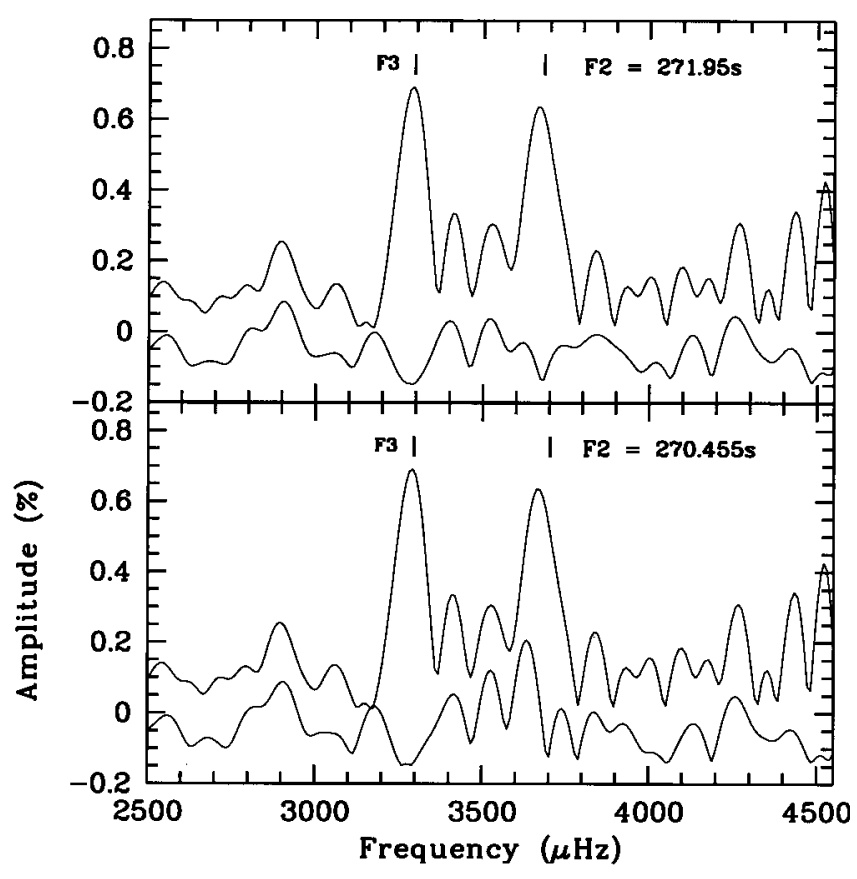

Fig. 4. Expanded view of the Fourier Transforms of the $5500 \AA$ light curve around the region containing F2 and F3. The lower curve in both panels shows the residuals (offset by $-0.15 \%$ ) as obtained by using the periods indicated for F2. The choice of $271.95 \mathrm{~s}$ for F2 provides a clearly better fit.

in each of the panels of Fig. 3). Therefore, even though Kepler et al. (1995) assigned a lower significance to the former than to the latter we chose to fix the period of this peak to $271.95 \mathrm{~s}$. We have checked that this choice is of no relevance to what follows. The amplitudes and phases of the modulations in each light curve are listed in Table 1.

Figure 3 shows that the periodicity at $215 \mathrm{~s}$ is clearly detected at all wavelengths, while those at 272 and $304 \mathrm{~s}$ are more difficult to discern in the ultra-violet datasets (Fig. 3). The phases for each of the modes are identical within the errors for the optical dataset. This is to be expected if the variations in brightness are dominated by variations in temperature (Robinson et al. 1982). This is not the case for the ultra-violet datasets presented here, as the data were not acquired simultaneously in the different passbands i.e. the phases in Table 1 are given with respect to the (different) zero points for each run.

From Fig. 3 and Table 1, it is clear that the behaviour of F2 is markedly different from that of either F1 and F3 in that its amplitude increases by more than expected if it were to be adequately described by a low spherical degree mode.

In order to check that any dependence on wavelength was not introduced by the way in which the light curves were constructed, we deselected the $-3500<t(\mathrm{~s})<-2500$ region of each of the lightcurves (see Fig. 2) - which shows somewhat similar modulations for the 4585 and $4210 \AA$ light curves - and fit these light curves in the manner described above. We found that the amplitude of F2 only changed by 0.02 , 0.04 , and $0.06 \%$ going from the longest to shortest wavelength light curves. These changes are insignificant compared to the
Table 1. Amplitudes and phases of the modulations derived from the optical and ultra-violet light curves.

\begin{tabular}{|c|c|c|c|c|}
\hline Mode & $\begin{array}{c}\text { Period } \\
(\mathrm{s})\end{array}$ & $\begin{array}{c}\text { Frequency } \\
(\mu \mathrm{Hz})\end{array}$ & $\begin{array}{l}A_{\mathrm{L}} \\
(\%)\end{array}$ & $\begin{array}{l}\Phi_{\mathrm{L}} \\
\left({ }^{\circ}\right)\end{array}$ \\
\hline \multicolumn{5}{|c|}{ Optical (5500 ̊̊) } \\
\hline $\mathrm{F} 1$ & 215.20 & 4646.9 & $2.01 \pm 0.13$ & $-153 \pm 4$ \\
\hline $\mathrm{F} 2$ & 271.95 & 3677.1 & $0.63 \pm 0.13$ & $107 \pm 12$ \\
\hline$(\mathrm{F} 2$ & 270.46 & 3697.5 & $0.58 \pm 0.13$ & $109 \pm 13)$ \\
\hline F3 & 304.05 & 3288.9 & $0.96 \pm 0.13$ & $-55 \pm 8$ \\
\hline \multicolumn{5}{|c|}{ Optical (4585 Å) } \\
\hline $\mathrm{F} 1$ & 215.20 & 4646.9 & $2.37 \pm 0.23$ & $-152 \pm 6$ \\
\hline $\mathrm{F} 2$ & 271.95 & 3677.1 & $0.99 \pm 0.23$ & $94 \pm 13$ \\
\hline$(\mathrm{F} 2$ & 270.46 & 3697.5 & $0.77 \pm 0.23$ & $96 \pm 17)$ \\
\hline F3 & 304.05 & 3288.9 & $1.07 \pm 0.23$ & $-50 \pm 12$ \\
\hline \multicolumn{5}{|c|}{ Optical (4210 ̊) } \\
\hline $\mathrm{F} 1$ & 215.20 & 4646.9 & $2.50 \pm 0.37$ & $-153 \pm 8$ \\
\hline $\mathrm{F} 2$ & 271.95 & 3677.1 & $1.22 \pm 0.37$ & $97 \pm 17$ \\
\hline (F2 & 270.46 & 3697.5 & $0.83 \pm 0.37$ & $96 \pm 26)$ \\
\hline F3 & 304.05 & 3288.9 & $1.08 \pm 0.37$ & $-39 \pm 20$ \\
\hline
\end{tabular}

$U V ; v 3(1920 \AA)$

$\begin{array}{lllll}\text { F1 } & 215.20 & 4646.9 & 4.56 \pm 0.33 & -55 \pm 4 \\ \text { F2 } & 271.95 & 3677.1 & 1.61 \pm 0.33 & -20 \pm 12 \\ \text { (F2 } & 270.46 & 3697.5 & 1.72 \pm 0.33 & -16 \pm 11) \\ \text { F3 } & 304.05 & 3288.9 & 2.01 \pm 0.33 & -20 \pm 9\end{array}$

$U V ; v 4(1920 \AA)$

$\begin{array}{lllll}\text { F1 } & 215.20 & 4646.9 & 5.04 \pm 0.32 & 152 \pm 4 \\ \text { F2 } & 271.95 & 3677.1 & 2.00 \pm 0.32 & -128 \pm 9 \\ \text { (F2 } & 270.46 & 3697.5 & 2.27 \pm 0.32 & -124 \pm 8) \\ \text { F3 } & 304.05 & 3288.9 & 1.19 \pm 0.33 & -157 \pm 16\end{array}$

$U V ; v 1(1570 \AA)$

\begin{tabular}{lllll} 
F1 & 215.20 & 4646.9 & $9.51 \pm 1.34$ & $-128 \pm 8$ \\
F2 & 271.95 & 3677.1 & $1.43 \pm 1.34$ & $-66 \pm 54$ \\
F2 & 270.46 & 3697.5 & $1.25 \pm 1.34$ & $-95 \pm 62)$ \\
F3 & 304.05 & 3288.9 & $3.12 \pm 1.34$ & $-153 \pm 24$ \\
\hline
\end{tabular}

Notes. $A_{\mathrm{L}}$ and $\Phi_{\mathrm{L}}$ are the amplitudes and phases obtained by iterative least squares fitting of the light curves. The periods have been fixed to those determined from WET data (Kepler et al. 1995) and with a precision higher than that listed above: $\mathrm{F} 1=215.1968 \mathrm{~s}, \mathrm{~F} 2=271.95 \mathrm{~s}$, $\mathrm{F} 3=304.052 \mathrm{~s}$. As such, in the ultra-violet, the measurements above represent limits to the amplitudes at a given frequency when the signal is not clearly detected (see Fig. 3). Also shown in parenthesis, are the values obtained for the 270.46 s periodicity which was assigned a lower probability of being a false peak by Kepler et al. (1995). Limits for $119.84 \mathrm{~s}(8344.7 \mu \mathrm{Hz})$, a potential real mode reported by Kepler et al. (1995) are: v1 $4.08 \pm 1.34 \%$ v3 $0.72 \pm 0.33 \%$, v4 $0.94 \pm 0.32 \%$; optical (5500 $\AA$ ): $0.09 \pm 0.11 \%$. The "v" numbers merely denote abbreviated run numbers.

errors attached to the measurements which are nearly identical to those listed in Table 1.

In principle, a varying background could give rise to an additional wavelength-dependence in the light curves that may 


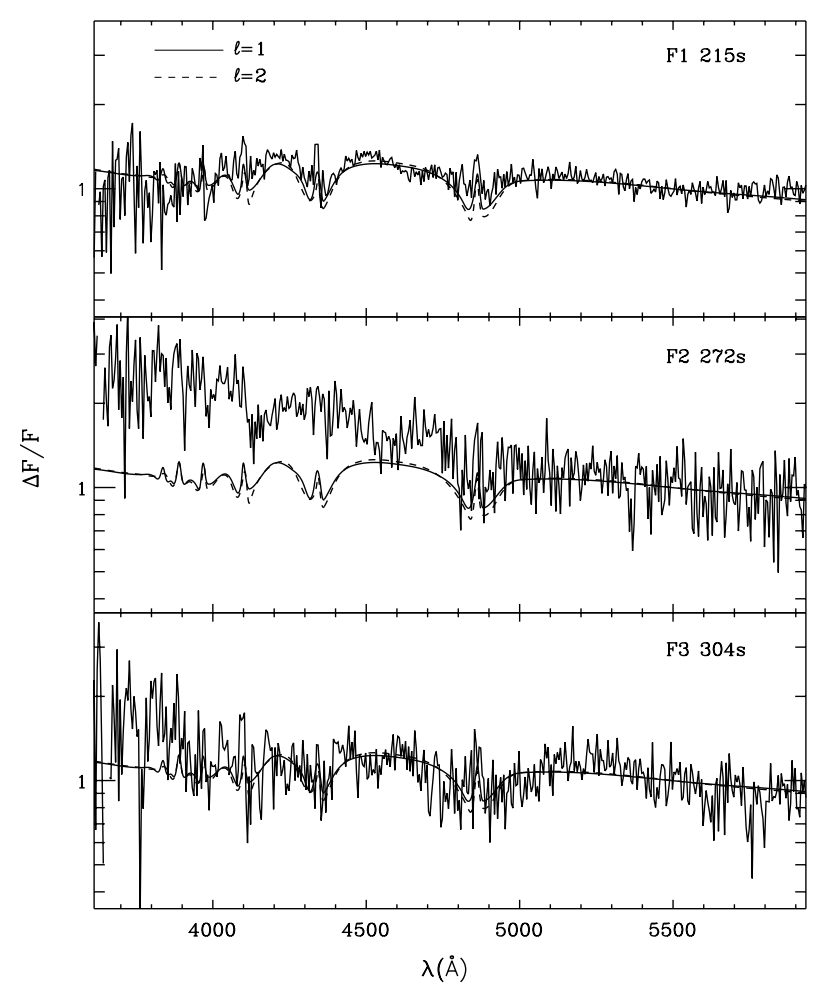

Fig. 5. Fractional wavelength dependent pulsation amplitudes of each of the 3 modes calculated in $5 \AA$ wide bins. The period is indicated next to the name. The observations have been overlaid with model chromatic amplitudes with $T_{\text {eff }}=11.5 \mathrm{kK}$ and $\log g=8$ with ML2 $/ \alpha=$ 0.6. Both the observations and the models have been normalised to unity at $5500 \AA$. The models have been convolved with a Gaussian function $(F W H M=4.4 \AA)$ to emulate a seeing profile. See Sect. 5.1 for details of the parameters used in computing the models.

have the effect of artificially enhancing mode amplitudes. To test this possibility, we constructed light curves of the background in each of the three passbands. We do not find any peaks in the Fourier Transforms of these light curves that are coincident with the modulations we find in G 117-B15A. We test this more rigorously in Sect. 5.2.

\section{Chromatic amplitudes and phases}

As mentioned earlier, the fractional wavelength-dependent pulsation amplitudes (i.e. "chromatic amplitudes") can be used to infer the $\ell$ value of a mode. The technique is based on the expectation that although all modes suffer cancellation due to integration over the stellar disc, this cancellation is diminished at shorter wavelengths due to limb-darkening, and is a function of the $\ell$ character of a mode. Thus, not only are the pulsation amplitudes larger at shorter wavelengths, but higher $\ell$ modes $(\ell \lesssim 3)$ can potentially be observed. At optical wavelengths, smaller effects within the Balmer lines are also apparent.

Chromatic amplitudes for F1, F2, and F3 are shown in Fig. 5. They were calculated by fitting the three periodicities in each $5 \AA$ bin, and by fixing the frequencies to those listed in Table 1, but leaving the amplitudes and phases free to vary. The resulting chromatic phases are shown in Fig. 6. Fixing the phases to the values in Table 1 makes almost no difference to

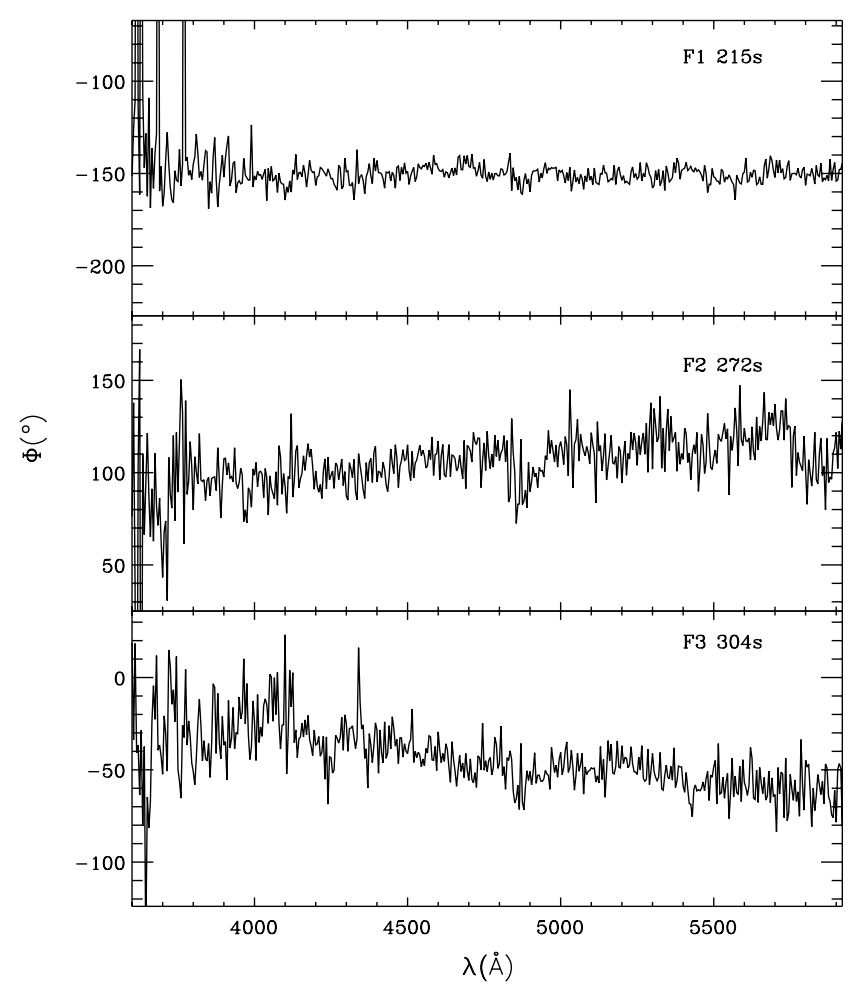

Fig. 6. Chromatic phases. The slopes in the continuum phases of F2 and F3 are unexpected. Based on simulations, however, we believe that this might not be intrinsic to the star. This, in contrast to the unexpected deviations from the models of the chromatic amplitudes for F2 (Fig. 5; see text).

the shape of the chromatic amplitudes, not even for F3, which shows a fairly large variation in phase.

Synthetic chromatic amplitudes, computed using model atmospheres kindly provided by D. Koester, and having $\ell=1$ and $\ell=2$ are overlaid in Fig. 5 for $T_{\text {eff }}=11.5 \mathrm{kK}$. Compared to these models, as well as chromatic amplitudes of other ZZ Cetis, that of F1 closely matches the models, while that of F2 shows a pronounced slope and a lack of distinct line cores; the chromatic amplitude of F3 is noisier and consistent with both $\ell=1$ and $\ell=2$ models. That $\mathrm{F} 2$ is rather different from $\mathrm{F} 1$ and $\mathrm{F} 3$ can also be appreciated from the Fourier transforms shown in Fig. 3, where it is much stronger at $4210 \AA$, relative to its strength at $5500 \AA$, than either F1 or F3. We discuss the constraints on spherical degree and effective temperature that can be placed using the chromatic amplitude of F1 in Sect. 5.1 below.

We now turn to the chromatic phases (Fig. 6). As mentioned earlier, the continuum phases are expected to be constant with wavelength. Although this is the case for F1, both F2 and F3 show large deviations - of the order of tens of degrees compared to $1-2^{\circ}$ found in previous studies (e.g. ZZ Psc, Clemens et al. 2000). We argue below (Sect. 5.2) that these variations are most likely due to noise. 


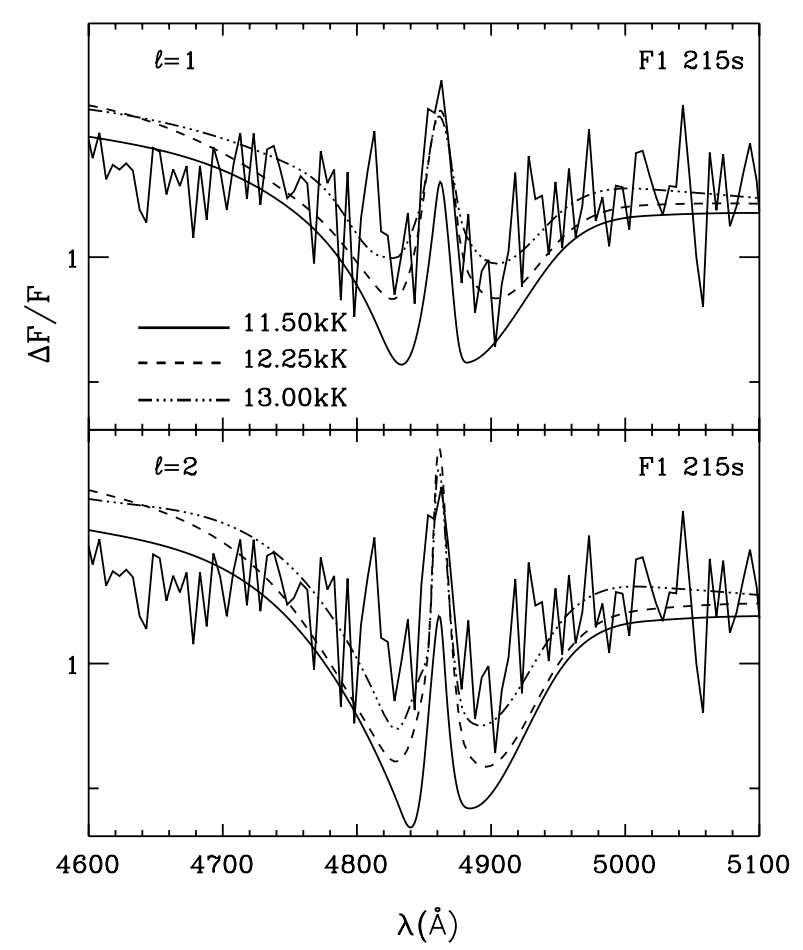

Fig. 7. Expanded view of the chromatic amplitudes and models around $\mathrm{H} \beta$. The top panel shows the strongest mode, F1, overlaid with models having $\ell=1$ and three different effective temperatures, as indicated. The bottom panel shows the same models for $\ell=2$. The $13.0 \mathrm{kK}$ model fails to match the observations for both $\ell$ values. The $\ell=1$ models are a better match at either of the other two effective temperatures considered than the $\ell=2$ models. This is apparent both in the wings and in the line cores. Note however, that for our grid of models, the $12.25 \mathrm{kK}, \ell=1$ model is a better match in the wings of the lines than the corresponding $11.5 \mathrm{kK}$ model.

\subsection{Spherical degree of $F 1$ and the effective temperature of $G$ 117-B15A}

Given the faintness of $\mathrm{G} 117-\mathrm{B} 15 \mathrm{~A}(V=15.54)$ relative to other objects which have been studied using this technique (e.g. ZZ Psc, $V=13.0$; Clemens et al. 2000), and the correspondingly lower signal-to-noise ratio, it is difficult to distinguish between $\ell=1$ and $\ell=2$ using the models in the continuum regions, even for the strongest mode (F1). However, the $\ell=1$ model is a better match in the wings of the line cores than the $\ell=2$ model (see Fig. 7). This is true even for models with different effective temperatures.

We can use the chromatic amplitude of F1 to constrain the effective temperature. This is useful, since model spectra of pulsating DA white dwarfs are not very sensitive to small changes in the atmospheric parameters, and fits to the average spectrum almost never yield unique results. This is because the Balmer lines reach their maximum strength in the ZZ Ceti instability strip. Other constraints, e.g. ultra-violet spectra or parallaxes are necessary to pin down the atmospheric parameters (Koester \& Vauclair 1996).

Bergeron et al. (1995) find that the optical and ultraviolet observations are best reproduced with the ML2 mixing length prescription, with the mixing length parameter $\alpha=0.6$.

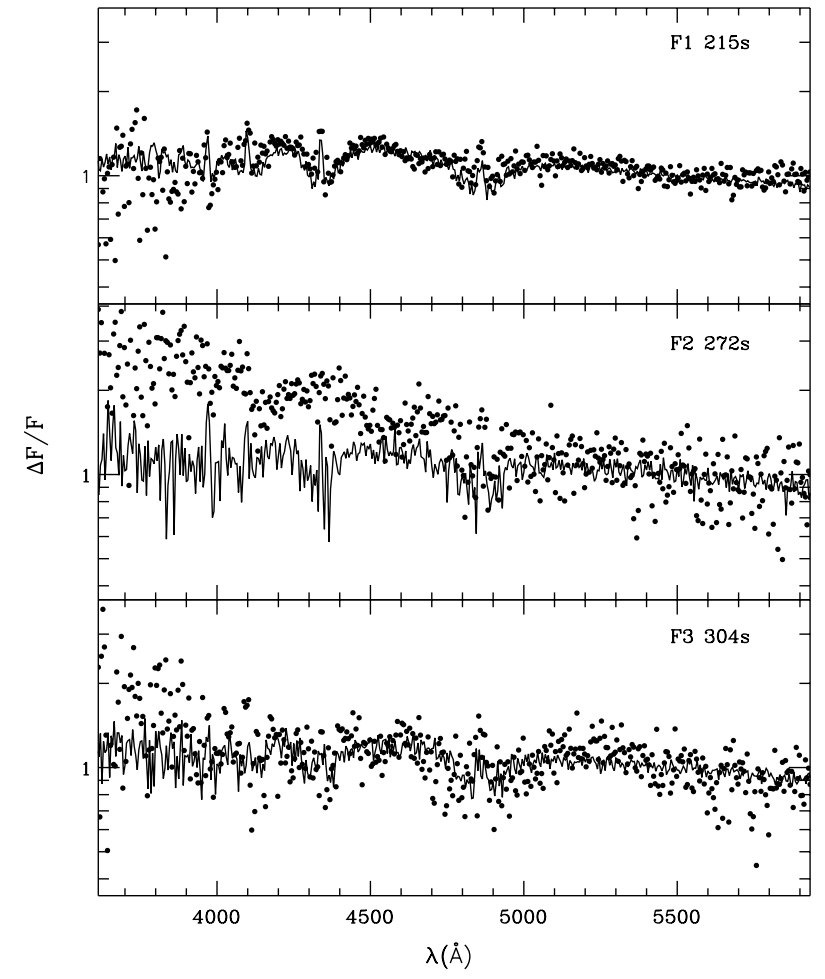

Fig. 8. Observed chromatic amplitudes (dots) and synthetic chromatic amplitudes (full line) with noise added and treated in exactly the same way as the data (see Sect. 4 for details).

For G 117-B15A, they infer $T_{\text {eff }}=11620 \mathrm{~K}$ and $\log g=7.97$. The models used here have also been computed using the above prescription. From fits (not shown) to the Balmer lines in our average spectrum, we infer $\sim 11750 \mathrm{~K}$, consistent with Bergeron et al. (1995). Our chromatic amplitudes of F1, however, can be reproduced better using a higher temperature, of $\sim 122250 \mathrm{~K}$. A similar difference was found for ZZ Psc by Clemens et al. (2000) and for the DBV, GD 358 by Kotak et al. (2003). This may indicate flaws in the model atmospheres or in the way we use them to calculate chromatic amplitudes. Below, we adopt $T_{\text {eff }}=11620 \mathrm{~K}$ where necessary, but stress that this choice has little bearing on what follows.

\subsection{Is the $272 s$ mode intrinsically weird?}

The chromatic amplitude of F2 is strikingly different from that expected. We carried out two tests in order to to assess the contribution due to noise to the chromatic amplitude of F2. We first generated synthetic chromatic amplitudes using model spectra and the observed light curve. This was done as follows: first, for every point in the $5500 \AA$ light curve, we determined a temperature by simple scaling, taking the mean temperature to be $11.62 \mathrm{kK}$. Next, we interpolated in a grid of model spectra (all with $\log g=8$ ) to find a simulated spectrum for this point, and added random, normally-distributed noise (adding more noise at shorter wavelengths). Finally, these simulated spectra were used to calculate chromatic amplitudes. An example is shown in Fig. 8 overlaid on the observations. Two points are evident: first, it is conceivable that the line cores can 
Table 2. Result of Monte Carlo simulation.

\begin{tabular}{ccc}
\hline \hline $\begin{array}{c}\text { Central } \lambda \\
(\AA)\end{array}$ & $\begin{array}{c}\text { fake 1 } \\
(\%)\end{array}$ & $\begin{array}{c}\text { fake 2 } \\
(\%)\end{array}$ \\
\hline 5500 & 51.5 & 53.4 \\
4585 & 1.8 & 1.2 \\
4210 & $<1$ & $<1$ \\
\hline
\end{tabular}

Notes. fake 1 and fake 2 refer to the fake signals inserted between $2000-3000 \mu \mathrm{Hz}$ and $5500-6500 \mu \mathrm{Hz}$ respectively. The second and third columns show the fraction of trials (out of 1000) that either artificial peak had an amplitude at least as large as that measured in the corresponding passband for F2.

be diminished or enhanced by random noise peaks. Secondly, normally-distributed measurement noise alone cannot reproduce the slope of F2.

The slope shown by the chromatic phases of F2 and F3 is not reproduced (in either direction) by our simulations and cannot therefore be due to noise alone.

The above test rests on the assumption that the noise in our data is normally distributed. This may not be the case. For instance, instrumental effects could lead to variations that are coherent with wavelength. Most effects we can think of would be multiplicative and grey, and would seem unlikely to cause the deviant chromatic amplitudes seen for F2. In principle, however, it may be possible to conceive of a noise signal, perhaps an additive one, that is much stronger in the blue. To test whether such signals are present, and whether they could lead to chromatic amplitudes such as those observed for F2, we performed another test, now using each of the three optical passbands as follows. We first injected two sinusoidal signals in the $5500 \AA$ passband with arbitrary phases (between 0 and $2 \pi$ radians) and frequencies randomly chosen to lie between $2000-3000 \mu \mathrm{Hz}$ and between $5500-6500 \mu \mathrm{Hz}$ which are regions free of any obvious real signal (see top panel of Fig. 3). Both amplitudes were chosen to be equal to that of F2 as listed in Table 1. Next, we inserted these two signals in the two other passbands (4585 $\AA, 4210 \AA$ ), with the same phase and frequency, but with the amplitudes scaled to the ratios measured for $\mathrm{F} 1$ i.e. $\mathrm{F} 1_{4585} / \mathrm{F} 1_{5500}=1.29$ and $\mathrm{F} 1_{4210} / \mathrm{F} 1_{5500}=1.36$. This allows us to establish whether or not random noise peaks can artificially enhance the amplitude at shorter wavelengths in a manner similar to that seen for $\mathrm{F} 2$. We repeated this procedure 1000 times per passband and measured the amplitudes of the fake signals in exactly the same way as described in Sect. 4 for the real data. If the anomalous amplitudes of F2 shortwards of $\sim 5000 \AA$ are due to noise, then we expect to find that the artificial peaks have an amplitude at least as large as that of F2 in a large percentage of the trials. The outcome of this test is shown in Table 2. The results for the $4585 \AA$ and $4210 \AA$ regions confirm that the measured amplitude of $\mathrm{F} 2$ is larger than can be expected from the contribution of noise alone. (For the $5500 \AA$ A passband, the values near 50\% merely reflect the fact that we inserted signals at the strength of $\mathrm{F} 2$ and that noise will almost as often decrease as increase this signal).

The absolute difference in phases between the $5500 \AA$ and $4210 \AA$ passbands is as large as that observed for the

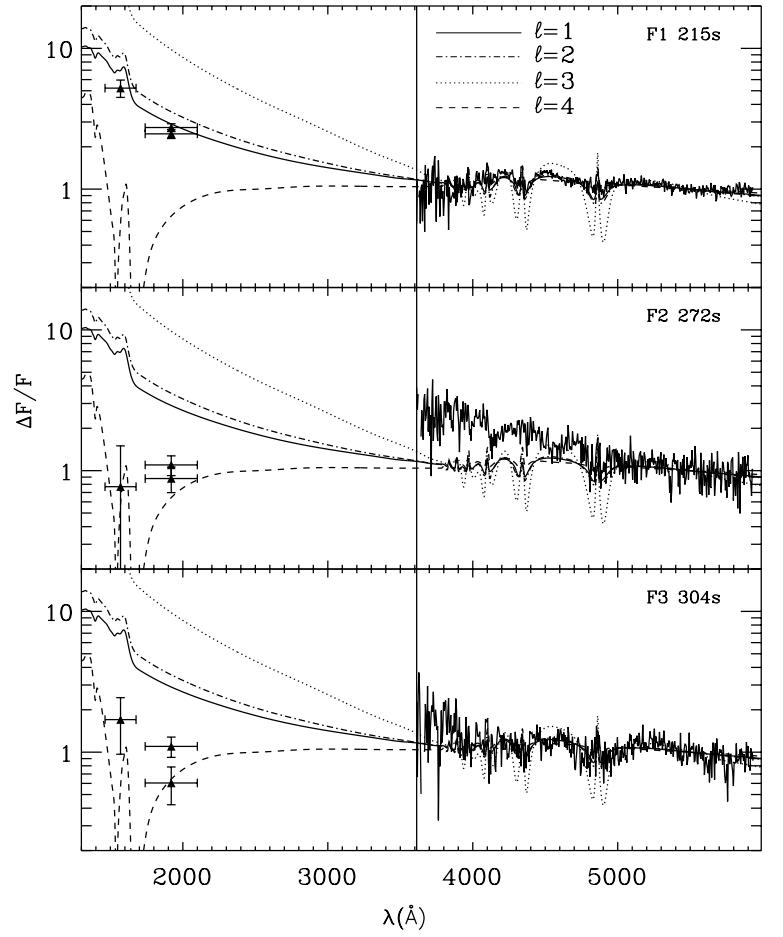

Fig. 9. Observed and model chromatic amplitudes for the whole wavelength range. The model shown here is the same as in Fig. 5 i.e. $T_{\text {eff }}=11.5 \mathrm{kK}, \log g=8$, and ML2 $/ \alpha=0.6$. The amplitudes have been normalised to unity at $5500 \AA$. The horizontal error bars on the ultra-violet amplitudes represent the $F W H M$ of the filters that were used. The vertical line merely serves to emphasise that the optical and ultra-violet observations are not contemporaneous.

chromatic phase of $\mathrm{F} 3\left(\sim 25^{\circ}\right) 13.6 \%$ and $7.6 \%$ of the trials for the peaks labelled fake 1 and fake 2 respectively. These values show that there is certainly some contribution due to noise to the slope of the chromatic phases of F2 and F3. The different contributions indicated by fake 1 and fake 2 probably reflect the fact that the noise level at the lower frequency end (fake1) shows a somewhat greater increase from $5500 \AA-4210 \AA$ than at the higher frequency end (fake2); this is seen in Fig. 3.

We conclude that the change in phase with wavelength shown by F2 may well be due to noise, but that the strange chromatic amplitudes are most likely intrinsic. F3 is simply too noisy to make a meaningful comparison.

\subsection{Constraints from the ultra-violet light curves}

Figure 9 shows the constraints obtained from ultra-violet photometry. Again, while F1 behaves as expected, i.e. larger amplitudes at shorter wavelengths, both F2 and F3 have smaller amplitudes in the ultra-violet than in the optical regions. For modes having $\ell \leq 2$, i.e. modes which are most likely to be observable, this behaviour is entirely unexpected. Model chromatic amplitudes for $T_{\text {eff }}=11.5 \mathrm{kK}$ show that only an $\ell=4$ model can match the data for F2 and F3.

As the optical and ultraviolet observations were not taken simultaneously, one might appeal to mode variability to explain the unexpected mismatch between optical and ultraviolet. For F3, we cannot exclude this possibility. For F2, however, 
the relative amplitudes in the ultraviolet, which were determined quasi-simultaneously, are also inconsistent with the predicted ones.

\section{Discussion}

In spite of the anomalous behaviour of F2, we continue, for the time being, to assume that this modulation seen in both the optical and ultra-violet light curves is due to pulsation. We base this assumption on the fact that the periods of F2 (272 s) and F3 (304 s) lie comfortably in the regime of non-radial $g$ mode oscillations, and that similar periods have been observed in several other ZZ Cetis. For instance, ZZ Ceti itself shows three dominant periods at 215, 271, and 304 s (Kepler et al. 1982).

We have only considered temperature variations when computing our synthetic chromatic amplitudes. It is well-known that these completely dominate all other sources of luminosity variations (Robinson et al. 1982). Any other variation would be expected to be independent of wavelength to first order. Also, any peculiar limb darkening law is at odds with those obtained from model atmospheres.

We briefly entertain the possibility that the that the $272 \mathrm{~s}$ modulation is actually a combination mode generated by real modes that are rendered invisible. This happens, for example, for the first harmonic of an $(\ell, m)=(1,0)$ mode as the inclination approaches $90^{\circ}$, and for the first harmonic of $(\ell, m)=$ $(2,0)$ mode at intermediate $\left(40-65^{\circ}\right)$ inclinations (see Fig. 2 in $\mathrm{Wu} 2001$ for $\ell=m$ modes or Fig. 4.9 in Kotak 2002 for $\ell \neq m$ modes). However, the flux variations are exceedingly low due to the cancellation suffered by high $\ell$ modes, even for a fortuitous inclination angle. As the slope of the chromatic amplitude in the optical cannot be explained by the above argument, this conjecture too must be discarded.

An intriguing possibility is that the $272 \mathrm{~s}$ mode is not a $g$-mode at all. The studies of Saio (1982) and Berthomieu $\&$ Provost (1983) showed that $r$-modes could be excited in $\mathrm{ZZ}$ Ceti type variables with periods similar to those of $g$-modes. However, to first order in $\Omega$ (the angular frequency of rotation), an $r$-mode produces no brightness changes (Saio 1982; Kepler 1984). An estimate of $v \sin i$ for G 117-B15A is not available, but other white dwarfs have been found to rotate with periods of about one day. Unless G 117-B15A is an exceptionally fast rotator, $r$-modes are probably do not give rise to F2. Estimates of the contamination of $g$-modes by $r$-modes cannot be established without detailed modelling, but it is difficult to see why these should be wavelength-dependent.

The nature of F2 unfortunately remains unexplained as all global effects must affect all modes. Either strong nonlinear processes may have to be invoked, or the description of modes based on a single spherical harmonic may have to be called into question.

We end by pointing out that the behaviour of F2 and F3 in the ultra-violet is not unique to G 117-B15A; the $141 \mathrm{~s}$ mode in G 185-32 also shows similar behaviour (Kepler et al. 2000b).
One other similarity between F2 and F3 and the 141 s mode of $\mathrm{G} \mathrm{185-32}$ is that they are the lowest amplitude modulations present in the spectrum. Simultaneous ultra-violet and optical spectroscopy might help to clarify the nature of the puzzling variations in G 117-B15A and G 185-32. Since both G 117-B15A and G 185-32 are relatively close to the blue edge, it might be relevant to establish whether such behaviour is peculiar to white dwarfs just entering the ZZ Ceti instability strip. Our findings highlight the need for caution and mode identification by several different methods. Indeed, given the above, any attempts to measure the rate of change of period or to constrain the core composition based on either the $272 \mathrm{~s}$ or $304 \mathrm{~s}$ modes may prove to be futile.

Acknowledgements. R.K. would like to sincerely thank H-G. Ludwig for his interest and many fruitful discussions. R.K. would also like to thank the Royal Commission for the Exhibition of 1851 for partial support. Based in part on observations made with the NASA/ESA Hubble Space Telescope, obtained from the data archive at the Space Telescope Science Institute. STScI is operated by the Association of Universities for Research in Astronomy, Inc. under NASA contract NAS 5-26555.

\section{References}

Bergeron, P., Wesemael, F., et al. 1995, ApJ, 449, 258

Berthomieu, G., \& Provost, J. 1983, A\&A, 122, 199

Bradley, P. A. 1998, ApJSS, 116, 307

Clemens, J. C., van Kerkwijk, M. H., \& Wu, Y. 2000, MNRAS, 314, 220

Finley, D. S., Koester, D., \& Basri, G. 1997, ApJ, 488, 375

Hauschildt, P. H., Allard, F., Ferguson, J., et al. 1999, ApJ, 525, 871

Kepler, S. O. 1984, ApJ, 286, 314

Kepler, S. O., Mukadam, A., Winget, D. E., et al. 2000a, ApJ, 534, L185

Kepler, S. O., Robinson, E. L., Koester, D., et al. 2000b, ApJ, 539, 379 Kepler, S. O., Robinson, E. L., Nather, R. E., \& McGraw, J. T. 1982, ApJ, 254, 676

Kepler, S. O., Winget, D. E., Nather, R. E., et al. 1995, Balt. Astr., 4, 221

Koester, D. 2002, A\&ARv, 11, 33

Koester, D., Allard, N. F., \& Vauclair, G. 1994, A\&A, 291, L9

Koester, D., \& Vauclair, G. 1996, White Dwarfs, ed. J. Isern, M. Hernanz, \& E. Garcia-Berro (Kluwer), 429

Kotak, R. 2002, Thesis, Lund Observatory, Sweden

Kotak, R., van Kerkwijk, M. H., \& Clemens, J. C. 2002, A\&A, 388, 219

Kotak, R., van Kerkwijk, M. H., Clemens, J. C., \& Koester, D. 2003, A\&A, 397, 1043

McGraw, J. T., \& Robinson, E. L. 1976, ApJ, 200, L89

Nather, R. E., Winget, D. E., Clemens, J. C., et al. 1990, ApJ, 361, 309

Oke, J. B., Cohen, J. G., Carr, et al. 1995, PASP, 107, 375

Richer, H. B., \& Ulrych, T. J. 1974, ApJ, 192, 719

Robinson, E., Kepler, S., \& Nather, E. 1982, ApJ, 259, 219

Robinson, E., Mailloux, T., Zhang, E., et al. 1995, ApJ, 438, 908

Saio, H. 1982, ApJ, 256, 717

Wu, Y. 2001, MNRAS, 323, 248 